\title{
The cerebrospinal fluid in acute leukaemia of childhood: studies with the Cytocentrifuge
}

\author{
D. I. K. EVANS, COLLEEN O'ROURKE, AND PATRICIA MORRIS JONES
}

From the Department of Haematology, Royal Manchester Children's Hospital, Pendlebury, and the Department $\vec{O}$ of Child Health, University of Manchester

SYNOPSIS The Cytocentrifuge enables a satisfactory cytological preparation to be made when the cells in the cerebrospinal fluid are normal in number or only slightly increased. A technique has $c$ been developed and the results of its use are described in 114 consecutive samples from 50 children with acute leukaemia, with and without involvement of the central nervous system.

Analysis of the results showed that $30 \%$ of the samples with a normal cell count contained leukaemic cells when examined by Cytocentrifuge. Only $74 \%$ of the samples with raised counts were found to contain leukaemic cells. Diagnosis of leukaemic meningitis based on changes in the cell count alone in cerebrospinal fluid is clearly unreliable, and the Cytocentrifuge enables a more precise assessment of the cerebrospinal fluid to be made.

It also appears that changes in the levels of protein and glucose in the cerebrospinal fluid of $\$$ leukaemic patients are not directly related to the presence of leukaemic cells but are the results of $\overline{0}$ changes in the cell count from whatever cause. There appears to be no value in measuring protein and glucose levels when monitoring the cerebrospinal fluid in acute leukaemia for evidence of early involvement of the central nervous system.

Involvement of the central nervous system is a common event in children with acute lymphoblastic leukaemia on maintenance therapy, and accounts for the ending of remissions in the majority of cases (Broder and Carter, 1972). Current protocols of treatment are assessing the value of prophylactic irradiation of the cerebrospinal axis and intrathecal drug therapy as means to prevent this complication. The presence of blast cells in the crebrospinal fluid of a patient with acute leukaemia must be taken as an indication of relapse. It is also a reminder that other areas may contain sequestered leukaemic cells, and that further spread may follow. The incidence rises as survival rates increase, and approximately half the patients showing meningeal relapse have bone marrow relapse at the same time (Broder and Carter, 1972).

The presence of only a few leukaemic cells in the cerebrospinal fluid must consequently be considered to terminate remission, and there is a need for a method of examining cerebrospinal fluid which will show the presence of these cells reliably and consistently. The usual method of preparing cerebrospinal fluid for cytological examination, eg, centrifugation and re-suspension of the cell button in albumin, cannot always demonstrate the exact cell type when the total cell count is normal or only slightly raised. The Cytocentrifuge ${ }^{1}$ is being used with increasing frequency for this purpose, and we report here our results of examination by Cytocentrifuge of 114 consecutive specimens from 50 children with acute leukaemia together with details of total cell counts and estimations of protein and glucose.

\section{Materials and Methods}

The samples of cerebrospinal fluid were received in polystyrene containers from a leukaemia clinic. Many were collected in the fasting state as the patients were undergoing simultaneous bone marrow aspiration under anaesthetic. The patients were children with acute leukaemia, with and without known meningeal involvement. Some had received prophylactic irradiation of the central nervous system or intrathecal methotrexate. Lumbar puncture was performed at regular intervals to monitor 
the cerebrospinal fluid in patients without symptoms, as well as for the treatment of central nervous system relapse. The sample was processed for protein, total cell count, and culture if indicated, and for cytology. A separate $0.5 \mathrm{ml}$ sample collected into fluoride-oxalate was used for glucose estimation.

Specimens were prepared for cytological examination with the Cytocentrifuge as follows.

A little oil (Whitemor oil, Manchester Oil Refinery Ltd) was spread round the hole of the filter paper to cover an area of approximately $1 \mathrm{~cm}$ diameter, and the filter paper, labelled slide, and chamber were inserted into the Cytocentrifuge head. The cerebrospinal fluid was mixed well and 9 drops $(0.6 \mathrm{ml})$ were placed in the chamber followed by 9 drops of Seitz-filtered AB serum. (Small aliquots of AB serum were stored in the deep freeze and thawed before use.) The head was balanced and the samples were spun at $1000 \mathrm{rpm}$ for 10 minutes. The slide, chamber, and filter paper were removed together from the head and placed on a flat surface with the slide down. The chamber was removed and any excess fluid was aspirated from the paper with a pipette. The slides were dried in air, fixed in methanol for five minutes, and stained by flooding the slide with phosphate buffer pH 6.4 twice, adding 5 drops of Giemsa, mixing and staining for 90 to 120 seconds. After washing in pH 6.4 buffer the slides were dried in air.

The oil serves to delay absorption of the cerebrospinal fluid into the paper while the cells are spur. onto the slide and gives a higher yield of cells. The AB serum has a similar but smaller effect on absorption, and helps to preserve cell morphology (Spriggs and Boddington, 1968). It is important not to overstain the preparation as otherwise fine nuclear details are obscured.

\section{Results}

One hundred and fourteen specimens were received from 50 children with acute leukaemia (47 acute lymphoblastic, two lymphosarcoma, and one acute myeloid). In 11 cases $(9 \%)$, seven of which had a cell count of less than 1 cell per $\mu$, the Cytocentrifuge preparation was unsatisfactory: too few cells were present, or the morphology of the few cells seen was distorted. In view of the low cell counts, most of these samples were probably normal. A minimum harvest of 10 cells was considered satisfactory, but the number of cells harvested varied from sample to sample. In spite of using a standard method, samples with 1 or fewer cells per $\mu$ l yielded from less than 10 to over 100 cells per slide. Prompt processing appears to reduce the number of failures. At least 100 cells could be counted in most cases where the cell count was raised. The results are shown in table $I$.

\begin{tabular}{lll}
\hline Cell Count & $\begin{array}{l}\text { Cytocentrifuge } \\
\text { Leukaemic Cells }\end{array}$ & No. of Cases \\
\hline Less than 5 per $\mu 1$ & None seen & $40(35 \cdot 1 \%)$ \\
Less than 5 per $\mu 1$ & Present & $17(14 \cdot 9 \%)$ \\
5 or more per $\mu 1$ & None seen & $12(10 \cdot 6 \%)$ \\
5 or more per $\mu 1$ & Present & $34(29 \cdot 8 \%)$ \\
Less than 5 per $\mu 1$ & Inadequate & $11(9.6 \%)$ \\
& preparation & $114(100 \%)$ \\
\hline
\end{tabular}

Table I Total cell count and Cytocentrifuge findings in 114 samples of cerebrospinal fluid from 50 children with acute leukaemia

Forty samples $(35 \%)$ had a normal cell count and showed no leukaemic cells with the Cytocentrifuge. They are classed as normal. Thirty-four samples had both a raised cell count (five or more cells per $\mu l)$ and showed leukaemic cells in the Cytocentrifuge. They are classed as positive. The cell counts in this group ranged from 6 to 2400 per $\mu l$ and the proportion of leukaemic cells ranged from 2 to $95 \%$. We have assumed that any blast cells observed in these samples are of leukaemic origin. A typical example is shown in figure 1 .

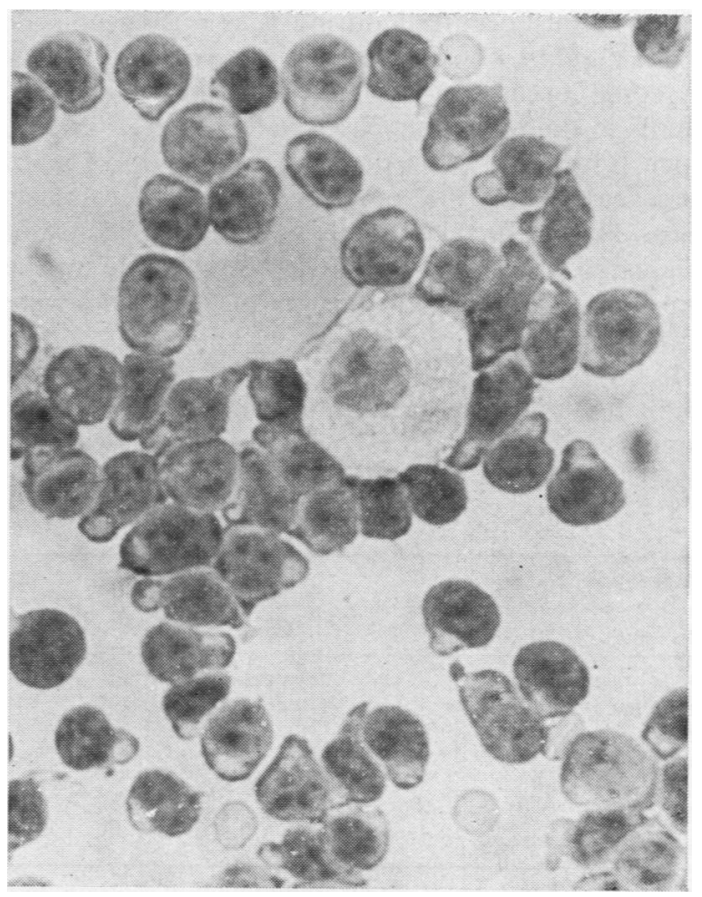

Fig 1 Leukaemic lymphoblasts and a histiocyte $(\times 900)$ 
Seventeen samples had a normal cell count (less than 5 cells per $\mu$ l) but were found to have leukaemic cells with the Cytocentrifuge. Ten of these cases had a cell count of only 1 per $\mu$ l or less. The proportion of leukaemic cells ranged from 2 to $39 \%$. Nine of these samples were from patients previously treated for meningeal involvement who would have been classed as successfully treated if the cell count were taken as the sole criterion. If these 17 are taken with the 34 cases classed as positive, there are 51 samples $(45 \%)$ with leukaemic cells in the cerebrospinal fluid and this group of 17 with a normal cell count yet with blasts in the cerebrospinal fluid by Cytocentrifuge comprises $33 \%$ of the total number with meningeal involvement. In other words, if a raised cell count had been taken as the only criterion of leukaemic meningitis, one third of affected cases would have been missed.

Twelve samples $(11 \%)$ had a raised cell count but showed no leukaemic cells with the Cytocentrifuge. The counts ranged from 6 to 50 cells per $\mu$ l. One of these cases had a cell count of $42 / \mu \mathrm{l}$ but had acute bacterial meningitis: another with a count of $50 / \mu l$ showed an unexplained pleocytosis in the cerebrospinal fluid with $97 \%$ neutrophils following a previous lumbar puncture at which leukaemic involvement had been found and treated with intrathecal methotrexate. This may have been due to infection or a reaction to therapy. Four further raised counts occurred during a remitting episode in one child in whom blast cells were clearly present on some occasions but not on others. The cell counts in the remaining cases were 10 per $\mu$ in one, 6 per $\mu l$ in three, and 5 per $\mu l$ in two. In only one of these cases was the raised count without blasts followed by a further sample with clear evidence of leukaemic involvement; and in four, including the sample with 10 cells per $\mu l$, subsequent samples were normal.

If this latter group of 12 samples with a raised count but no blasts is counted with the 40 normal samples, there is a total of 52 samples with no blasts found by Cytocentrifuge. Five of these samples, including the four samples from the one $\frac{0}{5}$ case previously mentioned, were found to have 을 meningeal involvement at a subsequent examina- $\vec{F}$ tion, and could therefore have been misdiagnosed. If these are excluded, there are 47 cases $(40 \%)$ with음 no meningeal involvement.

If this group of 12 cases is counted with the $34 \frac{\bar{\rho}}{\sigma}$ definitely positive cases, there are $46(40 \%$ of total $) \stackrel{\mathbb{D}}{\circ}$ samples with a raised cell count, of which 34 , ie, $74 \%$ \% of the positive samples, contained leukaemic cells. $\overrightarrow{0}$ Of the total 57 samples with a normal cell count, 17. (30\% of this apparently normal group) showed the $\vec{\omega}$ presence of leukaemic cells.

We also compared the glucose and protein levelso in these samples (table II). The samples with the higher total cell counts showed a significantly higher $\omega$ protein concentration $(P<0.025)$ but the presence $N$ of blasts was not significant $(\mathrm{P}>0.05)$. The differen- $\odot$ ces in glucose levels were less marked and the presence or absence of blasts again was not signifi- $\vec{z}$ cant $(P>0.05)$; but there was a statistically significant reduction of glucose levels in the samples withō raised cell counts from whatever cause $(P \simeq 0.025) . \vec{P}$ So changes in the cerebrospinal fluid protein and $\varphi$ glucose levels were not due to leukaemic involvement ${ }^{-}$ per se, but appeared to be secondary to the increase in cell count from any cause. There was no differences from normal when only a few leukaemic cells were present but the total cell count was not raised.

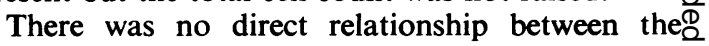
total cell count and protein levels in the patients $\vec{F}$ with a raised cell count, who only showed anక increase of protein as a group (fig 2). Nor was? there any relationship between protein and glucose levels in any of the groups of patients.

In some of the samples red cells were seen, due to? trauma at lumbar puncture, but these were spread? between all groups and did not influence either the number of cells counted or the total protein estimations.

The Cytocentrifuge also permits a differentiab count of the cells normally present in the cerebro $\frac{7}{0}$ spinal fluid. In the $\mathbf{4 0}$ samples classed as normal the

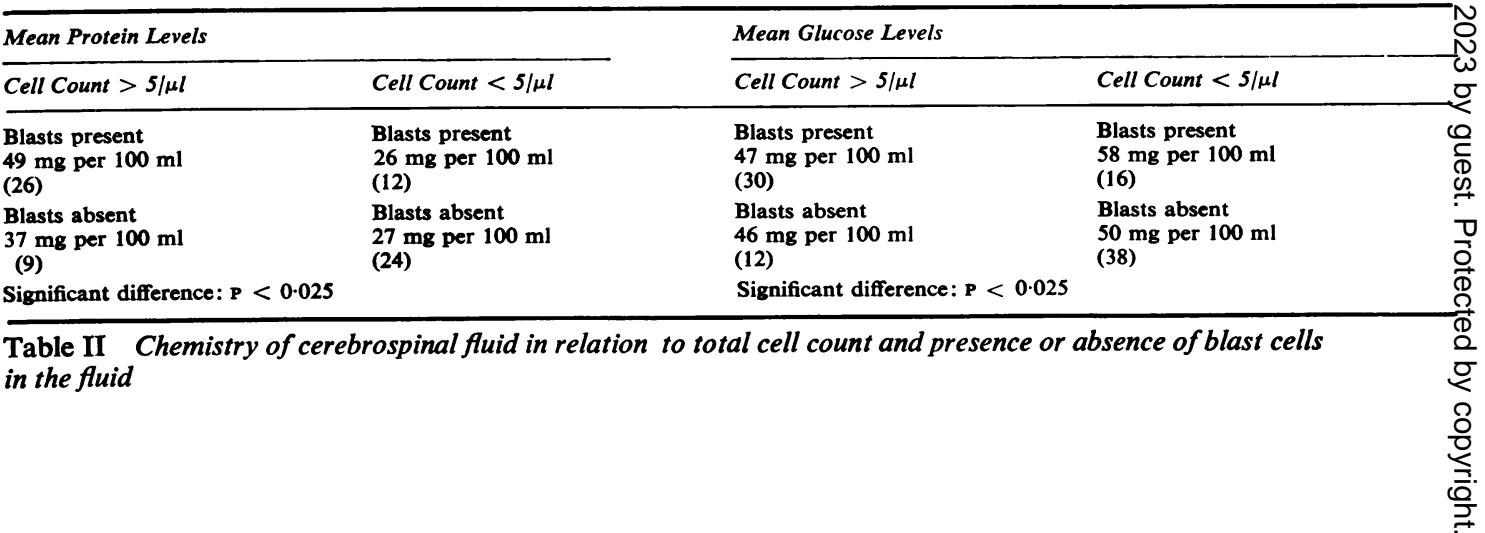




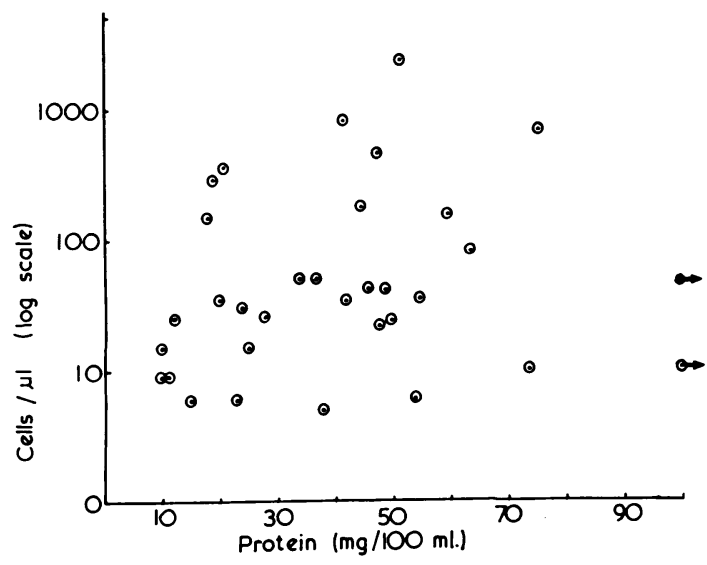

Fig 2 Cerebrospinal fluid protein levels in samples with a raised cell count.

average differential count was as follows: lymphocytes $44 \%$, histiocytes $34 \%$, neutrophils $9 \%$, and smear cells + other distorted cells $13 \%$. Lymphocytes were present in $38(95 \%)$ of these samples, histiocytes in $30(75 \%)$, neutrophils in $20(50 \%)$, and smear + other degenerate cells in $13(33 \%)$ (table III). Many of these children, although having no evidence of central nervous system leukaemia, had been treated by irradiation of the

\begin{tabular}{rll}
\hline Percentage & Cells Seen & $\begin{array}{l}\text { No. of Samples Showing } \\
\text { These Cells }\end{array}$ \\
\hline 44 & Lymphocytes & $38(95 \%)$ \\
34 & Histiocytes & $30(75 \%)$ \\
9 & Neutrophils & $20(50 \%)$ \\
13 & Smear cells + other & $13(33 \%)$ \\
& distorted cells & \\
\hline
\end{tabular}

Table III Distribution of cells in 40 samples of cerebrospinal fluid from children with acute leukaemia without central nervous system involvement

cerebrospinal axis or intrathecal drug therapy, and their cerebrospinal fluid may have been altered by these treatments. It is usually stated that the cells present in normal cerebrospinal fluid are lymphocytes, yet it is clear that neutrophils and histiocytes too may frequently be present. There is a possibility that these percentages overestimate the number of neutrophils and histiocytes as such cells may adhere better to the slide than the lymphocytes and hence give a falsely high figure. Similar proportions, with the addition of leukaemic cells, were seen in the samples from the patients with leukaemic meningitis. The presence of histiocytes (fig 3 ) has been previously noted (Spriggs and Boddington, 1968).

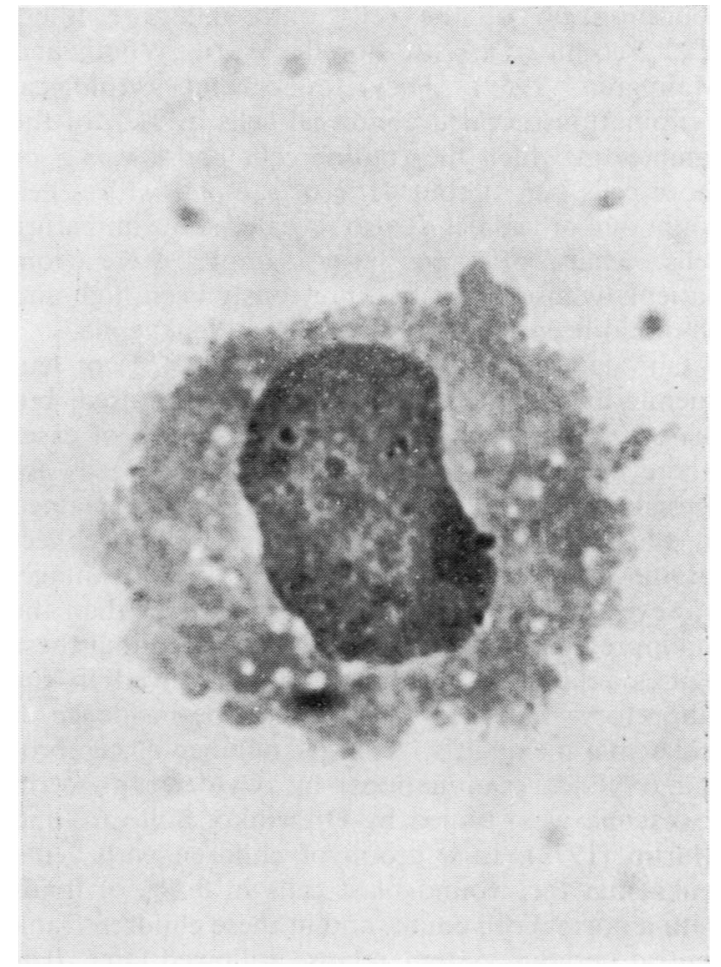

Fig 3 Histiocyte in normal cerebrospinal fluid $(\times 2000)$.

\section{Discussion}

Cells are always lost with the Cytocentrifuge. Dayan and Stokes (1973) estimated that only $15-30 \%$ are left on the slide after centrifuging, fixing, and washing. The method described here does not improve greatly on this figure, but gives a higher yield than when cerebrospinal fluid is used without the addition of serum and oil which increases the yield of cells by delaying absorption into the filter paper.

Morphology of cells processed by the Cytocentrifuge is sometimes unusual: leukaemic lymphoblasts may show marked nuclear distortion and indentation as the centrifugal force spreads the cells out on the slide in a manner not usually seen when blood or bone marrow smears are prepared in the usual way. But the cells are spread out thinly and details of nucleus and cytoplasm are easily recognized.

A comprehensive study of central nervous system leukaemia was reported by Nies, Malmgren, Chu, Del Vecchio, Thomas, and Freireich, in 1965. These authors examined 393 specimens from 78 patients with acute leukaemia, using a method involving 
concentration of the cells onto millipore filters (Del Vecchio, DeWitt, Borelli, Ward, Wood, and Malmgren, 1959). They found that cytological examination revealed abnormal cells in $94 \%$ of the samples in which the routine cells count was 5 or more cells per $\mu \mathrm{l}$; but $41 \%$ of samples with a cell count of 4 or less per $\mu$ l also revealed some immature cells. Many of these latter samples were from patients whose count had previously been high and who had been treated for meningeal leukaemia.

Our data show a lower incidence (74\%) of leukaemic involvement where the count is raised, but there is a similarly high proportion ( $30 \%)$ of cases where the count is normal but leukaemic cells are present. These differences may be partly explained by the fact that the Cytocentrifuge fails to collect all the cells in the sample. It has the advantage, however, of being more rapid and simpler than the millipore filter method. It should be noted that these figures refer only to the samples received in the laboratory: they do not represent the incidence of leukaemic meningitis. A larger number of cerebrospinal fluid examinations by Cytocentrifuge in leukaemia was studied by Drewinko, Sullivan, and Martin (1973). In a group of children with acute leukaemia they found blast cells in $8.5 \%$ of fluids with a normal cell count, and in these children frank central nervous system relapse followed later. It is clear that a normal cell count by itself is not sufficient to exclude disease of the central nervous system.

It is generally agreed that a count of 5 or more cells per $\mu \mathrm{l}$ in the cerebrospinal fluid is abnormal. Although we found some apparently normal cases with a higher count, our results do not suggest that any other figure would be a more reliable indicator of involvement of the central nervous system. The level should certainly be no higher.

Protein and glucose estimations on the cerebrospinal fluid give no relevant information in the individual case in which meningeal involvement is suspected; these tests are probably unnecessary. Although there is a higher protein level in the cases with a high cell count and blast cells present, it is the increase of cells which appears to lead to the increased protein level and not the leukaemic cells. Glucose levels in all the samples were on the low side as most were taken with the patient fasting. There is a significant reduction of glucose levels in the samples with higher cell counts; but again this is not an effect of the leukaemic cells but appears to be the effect of the raised cell count from any cause.

We conclude that any marked change in the level of protein or glucose in the cerebrospinal fluid of a patient with leukaemia should not be attributed to leukaemic infiltration of the central nervous system without excluding other possible causes.

We thank the Leukaemia Research Fund for support, and the Research Sub-Committee of the Royal Manchester Children's Hospital for the Cytocentrifuge. We are grateful to $\mathrm{Mr}$ M. Palmer for statistical assistance.

\section{References}

Broder, L. E., and Carter, S. K. (1972). Meningeal Leukaemia. Plenum Press, New York and London.

Dayan, A. D., and Stokes, M. I. (1973). Rapid diagnosis of encephalitis by immunofluorescent examination of cerebrospinal fluid cells. Lancet, 1, 177-179.

Del Vecchio, P. R., DeWitt, S. H., Borelli, J. I., Ward, J. B., Wood, T. A., Jr., and Malmgren, R. A. (1959). Application of millipore filtration technique to cytologic material. J. nat. Cancer Inst., 22, 427-431.

Drewinko, B., Sullivan, M. P., and Martin, T. (1973). Use of the cytocentrifuge in the diagnosis of meningeal leukaemia. Cancer (Philad.), 31, 1331-1336.

Nies, B. A. Malmgren, R. A. Chu, E. W. Del Vecchio, P. R. Thomas, L. B., and Freireich, E. J. (1965). Cerebrospinal fluid cytology in patients with acute leukaemia. Cancer (Philad.), 18, 1385-1391.

Spriggs, A. I., and Boddington, M. M. (1968). The Cytology of Effusions: Pleural, Pericardial and Peritoneal of Cerebrospinal Fluid, 2nd ed. Heinemann, London. 